\title{
Percepción de las Enfermeras frente al manejo del dolor en niños en un Servicio Oncológico Hospitalario en Chile
}

\section{Nurses' perception of pain management in children in a Hospital Oncology Service in Chile Percepção de enfermeiras sobre o manejo da dor em crianças em um Serviço Hospitalar de de Oncologia no Chile}

\author{
Denisse Parra Giordano ${ }^{1}$, ORCID 0000-0002-9439-2679 \\ Valentina Cuadro Maturana ${ }^{2}$, ORCID 0000-0002-8557-5182 \\ Romina Nies Moraga ${ }^{3}$, ORCID 0000-0001-6633-1724 \\ Constanza Pávez Castro ${ }^{4}$, ORCID 0000-0003-1869-9203 \\ Martina Romero Torres ${ }^{5}$, ORCID 0000-0003-1031-1497 \\ Consuelo Seoane Cabezas ${ }^{6}$, ORCID 0000-0002-2659-8253 \\ ${ }^{1}$ Departamento de Enfermería, Universidad de Chile \\ Escuela de Enfermería, Universidad de Chile
}

Recibido: 07/04/2020

Aceptado: 07/09/2020

Resumen: El manejo inadecuado del dolor en niños continúa siendo un problema. El dolor es un síntoma frecuente en los niños hospitalizados, siendo un gran desafío para enfermería, entendiendo que el dolor infantil en cáncer es multifactorial lo que dificulta su manejo. El objetivo fue conocer la percepción de los profesionales de enfermería frente al manejo del dolor en personas oncológicas pediátricas hospitalizadas durante el segundo semestre del año 2017. Metodología: Esta investigación se enmarca en el paradigma constructivista con enfoque en la teoría fundada. El estudio se realizó en el Servicio de Hemato-Oncología del Hospital Roberto del Río. Participaron seis enfermeras en una entrevista semiestructurada para un posterior análisis de contenido. Resultados: Fueron participantes de sexo femenino, adultos jóvenes y para la mayoría, esta constituye su primera experiencia laboral. El análisis cualitativo permitió estructurar la percepción del manejo del dolor en cuatro categorías con subcategorías: Definición de dolor: Concepto de dolor, Dolor en el servicio, Relevancia del dolor. Valoración del dolor: Subjetividad en la valoración, Aplicación de escalas de valoración, Experiencia personal, Rol familiar en valoración del dolor. Planificación de los cuidados: Rol de la familia en el manejo del dolor, Individualización de los cuidados, Trabajo en Equipo. Tratamiento del dolor: Tratamiento farmacológico, Tratamiento no farmacológico. Conclusiones: Se logró conocer la percepción de las enfermeras sobre el manejo del dolor, comprendiendo los niños y niñas como un ser multifactorial, que requiere una valoración adecuada, integrando el tratamiento farmacológico y no farmacológico para su remisión. 
Palabras claves: Manejo del Dolor; Enfermería; Niño; Oncología Médica; Dolor

\begin{abstract}
Inadequate pain management in children continues to be a problem. Pain is a frequent symptom in hospitalized children, being an excellent challenge for nursing, understanding that childhood pain in cancer is multifactorial, which makes its management difficult. The objective is to know the perception of nursing professionals regarding pain management in pediatric oncology people hospitalized during the second half of 2017. Methodology: This research is part of the constructivist paradigm focusing on grounded theory, descriptive with qualitative design. A study carried out at the Hemato-Oncology Service of the Roberto del Río Hospital proposed. Six nurses participate in a semi-structured interview for subsequent content analysis. Results: They are female subjects, mainly young adults and for the majority this constitutes their first work experience. The qualitative analysis allowed structuring the perception of pain management in four categories with subcategories: Definition of Pain: Concept of Pain, Pain in the service, Relevance of Pain. Pain assessment: Subjectivity in assessment, Application of rating scales, Personal experience, Family role in pain assessment. Care planning: Role of the family in pain management, Individualization of care, Teamwork. Pain management: Pharmacological treatment, Non-pharmacological treatment. Conclusions: It is possible to know the nurses' perception of pain, understanding boys and girls as a multifactorial being, which requires an adequate assessment, integrating pharmacological and non-pharmacological treatment for remission.
\end{abstract}

Keywords: Pain Management; Nursing; Child; Medical Oncology; Pain

Resumo: O tratamento inadequado da dor em crianças continua a ser um problema. A dor é um sintoma frequente em crianças hospitalizadas, sendo um grande desafio para a enfermagem, por compreender que a dor na infância no câncer é multifatorial, o que dificulta seu manejo. O objetivo é conhecer a percepção dos profissionais de enfermagem sobre o manejo da dor em oncológicos pediátricos internados no segundo semestre de 2017. Metodologia: Esta pesquisa está enquadrada no paradigma construtivista com foco na teoria fundamentada, descritiva com desenho qualitativo. O estudo foi realizado no Serviço de Hemato-Oncologia do Hospital Roberto del Río. Seis enfermeiros participam de uma entrevista semiestruturada para posterior análise de conteúdo. Resultados: São sujeitos do sexo feminino, principalmente jovens adultos e, para a maioria, constituem sua primeira experiência de trabalho. A análise qualitativa permitiu estruturar a percepção do manejo da dor em quatro categorias com subcategorias: Definição de dor: Conceito de dor, Dor no serviço, Relevância da dor. Avaliação da dor: subjetividade na avaliação, aplicação de escalas de avaliação, experiência pessoal, papel da família na avaliação da dor. Planejamento do cuidado: Papel da família no manejo da dor, Individualização do cuidado, Trabalho em equipe. Tratamento da dor: tratamento farmacológico, tratamento não farmacológico. Conclusões: É possível conhecer a percepção dos enfermeiros sobre o manejo da dor, compreendendo meninos e meninas como um ser multifatorial, o que requer uma avaliação adequada, integrando o tratamento farmacológico e não farmacológico para remissão. 
Palavras chave: Manejo da Dor; Enfermagem; Criança; Oncologia; Dor

Correspondencia: Denisse Parra Giordano, e-mail: denisseparrag@gmail.com

\section{Introducción}

El manejo inadecuado del dolor en niños continúa siendo un problema a nivel mundial (110). El dolor es un síntoma frecuente en los niños hospitalizados, siendo un gran desafío para enfermeras y enfermeros (2-6,8-14). El dolor infantil en cáncer es multifactorial dificultando su manejo $(4,15)$, pudiendo ser causado por la enfermedad de base o por procedimientos diagnósticos o tratamientos $(1,7)$. Así, en Chile un estudio plantea que la mitad de los niños hospitalizados refieren dolor durante su hospitalización, siendo la causa máxima de dolor las punciones con agujas y otros procedimientos médicos (14).

Cada niño es una totalidad histórica, construyendo su propia experiencia sobre dolor tanto física como emocional (2). De esta forma, configura su expresión de salud enfermedad entendido como el mismo proceso con carácter biológico y social (16).

El manejo del dolor comprende todas las actividades realizadas para la recuperación y disminución de la experiencia dolorosa. Según la Organización Mundial de la Salud (OMS), el alivio del dolor por cáncer es un problema de derechos humanos (17), por lo que su manejo es prioritario en el quehacer de enfermeras y enfermeros. El alivio del dolor y sufrimiento se considera el pilar de los derechos del paciente, y entonces una responsabilidad esencial del desarrollo de enfermeras y enfermeros (2). Enfermería es una profesión cuantiosa a nivel mundial (18), siendo su esencia el cuidado de las personas en todos los niveles de atención; que se desarrolla en condiciones de trabajo que, por lo general, implica falta de recursos y alta demanda laboral (19).

El primer paso en el manejo del dolor es su valoración, la cual permite realizar una intervención de enfermería personalizada integral, logrando disminuir y evitar el dolor (2). Para la detección y tratamiento del dolor, es fundamental considerarlo como una quinta constante vital, con el fin de objetivar el proceso, y así definir el manejo más adecuado (2). De esta manera, pueden presentarse diferentes formas de evaluarlo: autoinforme, observación y medidas fisiológicas (7).

Esta multifactorialidad en la valoración del dolor está dada por la edad, el desarrollo neurológico, diferencias individuales en la percepción y expresión de este, contexto de la situación dolorosa y experiencias previas personales $(1,4,9)$; igualmente, de factores socioculturales que modulan la percepción del dolor, como el sexo, conciencia de la posición social, identidad y expectativa de terceros $(6,20)$. Es por esto que la OMS hace hincapié en que no existe un instrumento de medición adecuado para todas las edades o tipos de dolor, por lo cual surge la necesidad de utilizar instrumentos adaptados según el ciclo vital, cultura y desarrollo, y de esta manera, ser utilizado sistemáticamente $(6,20)$.

Actualmente existe una amplia gama de herramientas para evaluar el dolor, y frente a la naturaleza del dolor causado por cáncer, su intensidad es útil por sí sola para la realización de la valoración en niños con cáncer (15). 
Por otro lado, el profesional de salud tiene a su disposición diferentes escalas para evaluar las características del dolor, en cuanto a localización, tipo, duración, frecuencia, intensidad, irradiación, síntomas y signos acompañantes, factores agravantes, factores atenuantes y medicamentos que alivian o provocan el dolor $(1-3,8,15)$, junto con el uso de escalas multidimensionales (15). La participación de la madre y niño en la valoración, la presencia del equipo de salud en el proceso y el apoyo de las enfermeras mejoran de manera importante el manejo del dolor en niños hospitalizados (5).

Las enfermeras refieren tener conocimiento para evaluar el dolor, y confiar en el autoinforme de dolor de los niños (planteando enfoques multidimensionales que este considere como indicadores asociados a la experiencia dolorosa), el reporte de los padres, y el criterio y competencia de las enfermeras para evaluar el dolor $(11,15)$.

Particularmente, el tratamiento por cáncer infantil transcurre en un largo período en el que el contacto diario entre enfermeras y la persona enferma va más allá de conocimientos técnicocientíficos, ya que considera también, el acompañamiento y una preparación emocional para que el niño y su familia lleven de mejor manera el proceso (21). El principal tratamiento del dolor pediátrico en oncología son los opiáceos, pese a la resistencia aún presente en su utilización (13), se suma a esto medidas no farmacológicas, las que resultan primordiales para entregar un manejo integral del dolor en el niño $(4,9)$.

En el manejo del dolor es imprescindible identificar los facilitadores de este proceso, dentro de los que destaca: apoyo de madre y niño; presencia del equipo de salud junto a la cama de los niños; presencia de los padres; buena relación entre enfermeras y niños, cuidadores y médicos; explicaciones del proceso dadas por enfermeras (5). Es importante considerar que las enfermeras refieren un mejor manejo del dolor que el evidenciado, y los padres son reacios a informar el dolor de sus hijos (12).

También es imprescindible determinar las barreras en el manejo del dolor, pues puede llevar al infra tratamiento, y así, ocasionar consecuencias fisiológicas y psicológicas negativas; debiendo las enfermeras superar estas dificultades para evaluar el dolor en niños hospitalizados $(1,10,11)$.

Como barreras se identifican la incorrecta valoración del dolor y sus características, creer que al tratar el dolor se enmascaran síntomas, falta de conocimientos sobre analgésicos y sus coadyuvantes, miedo a efectos adversos de analgésicos, falta de uso de técnicas cognitivoconductuales, edad del niño e información que entrega, y sobre todo carencia de recurso tiempo para desarrollar el proceso $(1,2)$. En el contexto de la organización, destacan las barreras de desequilibrio en la relación enfermera-paciente, falta de capacitación en el tema, falta de apoyo organizacional, falta de autonomía profesional y sensación de impotencia $(2,5,6)$.

La cultura afecta positiva y negativamente el manejo del dolor. No obstante, un fuerte liderazgo es fundamental para introducir innovaciones y llegar al manejo efectivo del dolor. Los aspectos claves para el manejo efectivo del dolor en niños son: liderazgo, recursos (tiempo, personal y dinero-fármacos) y confianza, esto implica disminución de estrés para todos, relaciones más confiables y mayor satisfacción laboral (12). Sobre las innovaciones necesarias en el manejo eficaz del dolor para mejorar la gerencia de este, se identifica como facilitador la entrega de confianza de las enfermeras, pues aumentan la confianza de niños y padres en su cuidado y disminuye la ansiedad; y como obstaculizadores, los recursos disponibles para el manejo del dolor $(2,6,12)$, así como el tiempo de capacitación del personal $(12,22)$.

El mejor manejo del dolor en niños implica mayores expectativas de niños y padres, lo que 
implica una presión positiva sobre las enfermeras para una gestión sistemática y efectiva del dolor (12). Frente a estos antecedentes, las enfermeras reconocen que, para empoderarse dentro del sistema organizativo hospitalario, es preciso un manejo efectivo del dolor y una entrega de cuidado de enfermería (6).

En este contexto, Jean Watson (1988) señala que las enfermeras deben ir más allá de los procedimientos, tareas y técnicas utilizadas en la práctica, entendiendo la salud, enfermedad y experiencia humana como un proceso de cuidado transpersonal, que requiere de un constante enriquecimiento personal y teórico, posicionando a la Enfermería como algo más profundo que la curación de una enfermedad (23). Por tanto, el rol de las enfermeras en el manejo del dolor se justifica en la gestión del cuidado humanizado y centrado en la persona, a partir de la adecuada valoración, intervención, seguimiento, prevención y reducción del dolor, en el que se involucren los valores, conocimientos, voluntad, y compromisos en la acción de cuidar (21), anteponiendo la preservación de la dignidad humana ante el modelo biomédico, que se condice con la filosofía y teoría del cuidado transpersonal de la enfermera desde Watson.

Al entender la enfermería como una profesión que se define histórica y socialmente, y entendiendo cómo las enfermeras que se desenvuelven en la Unidad de Hemato-Oncología manejan el dolor es necesario el enfoque constructivista entendiendo el proceso de salud enfermedad activo (16).

Para apoyar la comprensión de este fenómeno, y frente a la falta de evidencia específica sobre el manejo del dolor de enfermería en pediatría a nivel hospitalario en Chile, se plantea la siguiente investigación, la cual tiene por objetivo conocer la percepción de los profesionales de enfermería, frente al manejo del dolor en personas oncológicas pediátricas hospitalizadas.

\section{Material y Métodos}

La investigación corresponde a un estudio en el paradigma constructivista, enfoque teoría fundada, con análisis de contenido (24) como metodología analítica, lo cual permitió explorar el conjunto de expresiones verbales, junto a los diversos componentes que en él influyen. Se realizó en la Unidad de Hemato-Oncología del Hospital de Niños Roberto del Río (HNRR), establecimiento público de referencia nacional fundado en el año 1901, en Santiago de Chile. Se consideraron como participantes de la investigación a profesionales universitarias de enfermería que se desempeñaron durante el año 2017 en la unidad, contando con al menos un año de práctica profesional en dicho servicio o seis meses de experiencia en otras unidades y cuyo trabajo fuera exclusivo con niños oncológicos hospitalizados. De éstas, se excluyeron a quienes se encontraban con licencia prolongada o se mantuvieran fuera de sus labores por un tiempo igual o superior a una semana. Bajo estos criterios, la población susceptible corresponde a doce enfermeras en cuarto turno.

La información se obtuvo mediante entrevista semiestructurada, confeccionada por las investigadoras, previa revisión de la literatura. Se aplicó previamente a una enfermera de la unidad de cuidados paliativos del hospital para evaluar la atingencia de las preguntas. Finalmente, la entrevista incluyó antecedentes sociodemográficos y seis preguntas individuales, ¿qué entiende por dolor y cómo es éste en los niños hospitalizados del servicio?, ¿qué entiende por manejo del dolor?, ¿qué relación percibe entre el manejo del dolor y cuidado humanizado?, ¿cómo formulan los tratamientos para manejar el dolor y que integrantes del equipo realizan este proceso?, ¿cuál es su percepción sobre el proceso del manejo del dolor en el servicio? 
Las entrevistas se aplicaron por la misma investigadora a seis participantes (muestreo por conveniencia) hasta que el fenómeno fue conocido por las investigadoras (saturación). Las participantes concordaron en participar del estudio (no hubo abandono o desistir de las entrevistas) en una sala del Servicio con total privacidad, con una duración de 12 a 31 minutos, durante el segundo semestre del 2017. Las entrevistas fueron grabadas y posteriormente transcritas integralmente para orientar el análisis. Identificando a las entrevistadas con la letra " $E$ " y asignándoles un número según orden de realización, tomando fragmentos literales de los contenidos destacados agrupadas en la metacategoría del "Manejo del dolor", constituidas por categorías y subcategorías emergentes acordes al objeto de la investigación.

Para asegurar la validez de la investigación esta fue desarrollada según los criterios de rigor de Guba y Lincoln (25), realizándose triangulación de datos en grupos por las investigadoras; así como, se cuenta con la aprobación del Comité de Ética de Investigación en Seres Humanos de la Facultad de Medicina de la Universidad de Chile $\mathrm{N}^{\circ}$ 106-2017, cumpliendo así con los aspectos éticos de confidencialidad y la voluntariedad en la participación de las entrevistadas.

\section{Resultados}

Las personas entrevistadas fueron seis profesionales de enfermería. En relación con la caracterización sociodemográfica, todas las participantes fueron de género femenino, su rango etario fluctúa entre los 27 y 49 años, todas de nacionalidad chilena, para tres de ellas este servicio es su primera experiencia laboral y una tiene hijos como se muestra en Tabla 1.

Tabla 1: Caracterización sociodemográfica de las enfermeras entrevistadas (n=6). Santiago, Chile, 2017.

\begin{tabular}{lcc}
\multicolumn{1}{c}{ Características } & Número & Porcentaje (\%) \\
\hline $\begin{array}{l}\text { Sexo } \\
\text { Femenino }\end{array}$ & 6 & $100 \%$ \\
Edad & & \\
$21-30$ años & 4 & $66,6 \%$ \\
$31-40$ años & 1 & $16,6 \%$ \\
$41-50$ años & 1 & $16,6 \%$ \\
Posee hijos & 1 & $16,6 \%$ \\
Nacionalidad & & \\
Chileno & 6 & $100 \%$ \\
Extranjero & 0 & $0 \%$ \\
Experiencias laborales previas & 3 & $50 \%$ \\
\hline
\end{tabular}


Los fragmentos literales extraídos de las entrevistas se agruparon en la metacategoría "Manejo del dolor", entendido como las acciones terapéuticas realizadas con el fin de lograr la disminución o remisión del dolor, y que se compone de cuatro categorías, las que se describen a continuación. De esta emergen categorías y subcategorías como se muestran en la Tabla 2.

Tabla 2: Categorías y subcategorías desde metacategoría Manejo del dolor $(n=6)$. Santiago, Chile, 2017.

Categoría

1. Definición de dolor

2. Valoración del dolor

3. Planificación de los cuidados del dolor

4. Tratamiento del dolor

\section{Subcategoría}

Concepto de dolor

Dolor en el Servicio

Relevancia del dolor

Subjetividad en la valoración

Aplicación de escalas de valoración

Experiencia personal

Rol familiar en valoración del dolor

Rol de la familia en el manejo del dolor

Individualización de los cuidados

Trabajo en equipo

Tratamiento farmacológico

Tratamiento no farmacológico

\section{Categoría 1. Definición de dolor}

Se refiere a la construcción de la definición del dolor.

Concepto de dolor. Considerado como una experiencia personal, única y subjetiva, que no debe ser desestimada y que requiere de atención inmediata. Frente a esto, las enfermeras manifiestan que el dolor no solo afecta a quien lo padece, sino que también a su núcleo familiar.

"Experiencia única, personal, intransferible que se presenta cuando uno está expuesto a un agente dañino, por decirlo así, y que en realidad no solo nos afecta en lo físico, sino que puede ser un dolor más somático que también nos afecta en todos nuestros aspectos emocionales, psicológicos y también, por ejemplo, en el caso de aquí de los niños, afecta no solo a niños, sino que a todo su núcleo familiar." (E6)

Dolor en el servicio. En este ámbito, los factores estresores que afectan a los niños y niñas difieren de otros servicios. Por otro lado, introducen el concepto de "dolor del alma" el cual va más allá de lo físico, acompañado de un "dolor total", es decir, afecta todos los ámbitos de la vida por lo que requiere atención personalizada.

"Es como un "dolor total" (...) tiene muchos ámbitos que afectan "toda la vida del niño", por eso yo lo considero como "un dolor total" (E5)

Relevancia del dolor. Las enfermeras del Servicio de Oncología identifican al dolor como algo prioritario y plantean que todos los profesionales de la salud deben saber evaluar para 
valorarlo de manera obligatoria en su práctica clínica diaria, con el objetivo de darle una atención inmediata y oportuna, sin importar su intensidad:

"El dolor es algo prioritario, es algo que nosotros tenemos que aprender a evaluar, que tenemos que priorizar." (E5)

\section{Categoría 2. Valoración del dolor}

Constituye la evaluación de la experiencia dolorosa de los niños hospitalizados.

Subjetividad en la valoración. La experiencia dolorosa debe ser entendida según lo que refiere la persona, independiente de la percepción que se tenga como profesional.

"Lo que dice el niño, lo que expresa el niño es, independiente de que uno le haya pasado algo hace poco o que no tenga cara de que le duele, si el niño lo dice es, o sea acá está muy bien implementado eso, escalas y de que el dolor de verdad es una experiencia subjetiva y que en realidad nadie va a poder expresarlo, vivirlo, más que el niño." (E6)

Aplicación de escalas de valoración. Las enfermeras manifiestan que la evaluación se realiza con escalas validadas, destacando FLACC, EVA y ENA, cuya elección varía según la edad de la persona.

"Somos muy estrictos respecto a las escalas, respecto a cuál es la escala más adecuada para cada niño, cómo evaluarla, en qué momento evaluarla, acá en realidad el dolor es un signo vital más." (E6)

Experiencia personal. Destaca la importancia de las vivencias dolorosas personales en el manejo del dolor en la persona.

"El dolor es algo que nunca podemos olvidar. Porque yo creo que todos hemos tenido dolor en la vida, y es una experiencia que no se la damos a nadie. Todos los tipos de dolor, tanto físico como del alma. Entonces, encuentro que el tema que es tan tocando es super difícil, pero es super bonito a la vez, porque eeh, yo siento que del dolor salen las mejores cosas." (E5)

Rol familiar en valoración del dolor. Hace énfasis en que la participación de la familia es fundamental, dado a que comprenden el comportamiento de los niños y niñas a cabalidad, siendo su intervención una contribución a la objetivación de este.

"son ellos los que conocen al niño. No falta la mamá que dice "tía, como que está raro, como que algo le molesta, ¿usted cree que sea dolor?”, "ya probemos”." (E3)

\section{Categoría 3. Planificación de los cuidados del dolor}

Se refiere a la estructuración de las actividades a realizar en el manejo del dolor.

Rol de la familia en el manejo del dolor. Expone la importancia de la familia en el tratamiento farmacológico, dado que el equipo puede sugerir, pero ellos deciden finalmente su administración. Aún más destacable es que la familia actúa como potente contenedor emocional, siendo fundamental el tiempo de visitas, ya sea durante la hospitalización y en los procedimientos.

"la contención emocional que le brinda la familia es, pero yo creo que clave para el manejo del dolor, porque en el fondo tú le puedes poner muchos fármacos a un niño, pero si la familia no es contenedora, ese niño no va a estar tranquilo no más." (E1)

Individualización de los cuidados. Alude a la importancia de la evaluación personalizada, entregando los lineamientos para el tratamiento farmacológico En dicho proceso intervienen 
distintos profesionales; quienes se desempeñan en el servicio, el equipo de paliativos, e incluso el rol asistente social.

"Aquí no todos los niños son iguales, y no a todos se les puede tratar con los mismos fármacos ni con las mismas intervenciones." (E5)

Trabajo en Equipo. Refiere que la valoración del dolor es continua, donde todos los miembros del equipo de salud están capacitados para realizarla, destacando la participación del personal técnico de enfermería, por su mayor contacto con la persona hospitalizada.

"Todos, todos (con resalte de ojos, voz y manos), los técnicos cuando van a controlar los signos vitales valoran el dolor, ellas están capacitadas, nosotras claramente cada vez que vamos a ver al niño valoramos dolor y administramos tratamiento farmacológico, los médicos al prescribir todo lo que es la analgesia y las terapeutas complementarias que también vienen a hacerles las terapias de alivio del dolor". (E1)

\section{Categoría 4. Tratamiento del dolor}

Son los aspectos considerados para favorecer la recuperación y la disminución de la experiencia dolorosa.

Tratamiento farmacológico. Las entrevistadas coinciden en su importancia, destacando la práctica habitual del uso de opioides sin restricción, pese a observar dependencia asociada a su uso. Se hace énfasis en la importancia de la valoración y las experiencias de dolor de la persona, en la administración de la terapia. Se expone, además, que, pese a que el tratamiento farmacológico es una indicación médica, la decisión de cómo y cuándo administrarlo, es responsabilidad absoluta del profesional de enfermería.

"se les hace un esquema de terapia analgésica como según la OMS por lo general acá, y dependiendo de eso si es que se alivia bien...obviamente escala en esta en opiáceos ya cuando no cede mucho y se escala hasta que no haya dolor". (E1)

Tratamiento no farmacológico. Es fundamental para el correcto alivio del dolor, esto es, mediante escucha activa, acompañamiento, manejo ambiental y/o por medio de terapias complementarias; donde las enfermeras destacan el desarrollo de una relación terapéutica efectiva para un adecuado manejo del dolor. Por otro lado, hacen alusión al autoconocimiento como pilar fundamental en la entrega de cuidado humanizado, destacando la confianza y contención por sobre las técnicas y procedimientos.

"Cuando uno logra tener una relación terapéutica efectiva y eso es solo haciendo cuidados humanizados, van a tener plena confianza en ti y también que tú los vas a poder ayudar y eso les baja la carga de la ansiedad en el niño". (E1)

"... también tenemos en cuenta de que el manejo farmacológico no es todo respecto a cómo tratarlo, o sea uno cura con otras cosas también, cura con el toque, con un cariño, con una caricia y también eso está super claro." (E6)

\section{Discusión}

Tomando en consideración la caracterización sociodemográfica de los profesionales de enfermería de servicios oncológicos pediátricos, al igual que el presente estudio, en una investigación en Indonesia (6) prima el sexo femenino (90-100\%), donde sus edades fluctúan entre los 25 y 60 años, y los años de experiencia profesional previa varían según el complejo 
hospitalario, no existiendo un patrón definido al respecto. Asimismo, un estudio realizado en Brasil (26) permite visualizar una caracterización similar a la chilena, posicionando al sexo femenino como protagonista en los servicios de oncología, cuyas edades se mantienen entre los 25 y 50 años. Al contrario, en un estudio en Israel (11) las enfermeras pediátricas refirieron en promedio 9 años en el servicio pediátrico (10 en general), y la mayoría evalúa el dolor de los niños, utilizando sus propias impresiones, autoreporte del niño o el llanto de este, dependiendo de la personalidad de cada niño su cultura y umbral del dolor.

Estudio en enfermeras en Indonesia (6), refieren que en las salas de pediatría las enfermeras sentían que no eran capaces de brindar una atención eficaz contra el dolor a los niños hospitalizados; además el servicio aporta factores organizativos estructurales y culturales que dificultan la atención eficaz del dolor en pacientes pediátricos, integrándolos en la práctica clínica de las enfermeras; igualmente en Asturias-España, los conocimientos en el manejo del dolor son menores a la mitad de las enfermeras que trabajan con niños (22).

Respecto a la definición de dolor, en el presente estudio los resultados muestran que las enfermeras del servicio convergen en un mismo concepto de dolor, definición similar a la otorgada por la Asociación Internacional del Dolor (27), permitiendo unificar criterios en su manejo. En esta investigación, se reconoce que el concepto dolor se construye desde diferentes perspectivas, como se mantiene en estudio de Cuba (4) y Chile (28), entendiéndolo como una sensación total, en el cual influyen múltiples factores, requiriendo entonces un manejo dinámico y dependiente de las características de la persona y su entorno.

No obstante, en el servicio de oncología el dolor difiere de otras unidades, dado que las experiencias dolorosas no solo se atribuyen a la patología de base, sino que también, a su tratamiento, procedimientos y/o el enfrentamiento personal-familiar de la enfermedad.

$\mathrm{Al}$ igual que este estudio, una revisión bibliográfica mantiene que la relevancia del dolor como algo prioritario en cualquier atención, necesitando de valoraciones específicas (2), protocolizadas y bajo fundamento. Bajo estos antecedentes, las enfermeras concluyen que el manejo del dolor es un pilar fundamental en la calidad profesional, y que no solo debe visualizarse como una necesidad del paciente, sino como parte de un derecho fundamental (21).

En lo que concierne a la valoración del dolor, en pediatría la medición y valoración del dolor es considerada como una de las mayores dificultades, debido a la complejidad de una valoración completa para elegir las estrategias adecuadas $(2,9,10,29)$. Al igual que la presente investigación, en un estudio en Cuba las enfermeras comparten que el dolor es una sensación subjetiva, por lo que no puede ser cuestionada; siendo percibida de forma personal y única por cada persona (4) al no ser solamente una transmisión fisiológica sino también un valor subjetivo conforme a la edad, cultura, experiencias previas, y estado psicológico (1); es reconocida como una de las principales estrategias para el manejo del dolor su evaluación subjetiva (8).

La importancia de la utilización de escalas se mantiene a nivel internacional, ya que se evidencia su utilidad al aplicarse en función de la edad y desarrollo cognitivo de la persona (8). Las enfermeras del servicio en este estudio, perciben que las escalas de valoración del dolor se utilizan adecuadamente, al igual que un estudio realizado en Madrid-España (8); sin embargo, esta percepción se contrapone con un estudios en Cuba (17), que determinan que existe un déficit en la valoración por parte del profesional de enfermería, producto de la falta de comprensión en la aplicación de dichas escalas.

Respecto al conocimiento de estas, un estudio en Australia refirió que las enfermeras especializadas en pediatría están altamente capacitadas en conocimientos y actitudes para valorar 
el dolor (10), así también, un estudio en Madrid-España refiere que más de la mitad de las enfermeras están familiarizadas con las escalas de dolor (8). Sin embargo, en Israel (11) un 75\% informa que no las ha usado recientemente, y solo la mitad usa un método de valoración involucrando al niño; la mayoría confían en su propia impresión general y sólo un tercio informó que involucraba a los padres.

Como plantea la presente investigación, en la valoración del dolor se debe considerar la participación de la familia en el proceso, dado que son ellos quienes conocen y comprenden el comportamiento de los niños y niñas; entendiendo que la relación con los padres y las rutinas familiares son su relación segura con el mundo en estos primeros años (9).

Respecto a la planificación del dolor, al igual que este estudio, se plantea el involucramiento de la familia, y un trabajo en equipo, para mejorar su manejo; así como, la individualización de las intervenciones, con una pauta personalizada a su alta (1-3). Un estudio iraní (5) menciona, además del trabajo en equipo, la importancia de una comunicación adecuada, la relación amistosa entre enfermeras y pacientes, así como también, acompañantes y médicos, como el factor de éxito en el manejo del dolor, junto con el uso de intervenciones no farmacológicas; incluso un estudio español (22) refiere que la relación directa con la enfermera es la clave para el éxito del manejo eficaz en niños.

En relación al tratamiento del dolor se deben considerar dos aspectos, por un lado la anticipación a las experiencias dolorosas, y por otro, considerar el tratamiento multimodal al dolor (1). En el primer caso, se plantea el control cognitivo y conductual como una opción para el manejo del dolor en las intervenciones, previo a que el niño se vea enfrentado al dolor o angustia; es decir, las técnicas deben aplicarse antes de ingresar al examen o comenzar una técnica, identificando también actividades que hayan ayudado en el pasado (3).

En concordancia a lo planteado en este estudio, se presenta un tratamiento farmacológico asociado a la intensidad del dolor en el uso de la escalera analgésica (1) según la OMS (20) bajo indicación médica, sin embargo, las enfermeras destacan que son ellas las principales ejecutoras y responsables en su administración. No obstante, un estudio en Uruguay (30) detectó deficiencias en los conocimientos de los médicos respecto al tratamiento en el dolor en niños, principalmente en la prescripción de morfina.

Del mismo modo, en esta investigación se subraya la importancia de administrar la terapia sin temor a las dosis o a la posible adicción que esta conlleva, específicamente al uso de opiáceos, siendo importante tener claridad que no existe evidencia que el uso de analgésicos oculte los síntomas del dolor o dificulte su manejo, por lo cual, el costo o disponibilidad de estos no debe obstaculizar su uso $(7,29,30)$. Un estudio en Madrid-España (8) plantea la familiaridad en uso de opioides como una de las estrategias para mejorar el manejo del dolor, lo que demuestra una disminución -a nivel nacional- de la resistencia a su uso $(13,20)$ en la medida que su prescripción siga los lineamientos de protocolos basados en las recomendaciones de la OMS (31).

Finalmente, en este estudio se presenta el tratamiento no farmacológico, al igual que en España (1), se entiende que la ansiedad contribuye a la percepción del dolor, siendo esencial un ambiente tranquilo, la presencia de los padres y dar información al niño sobre el proceso; asimismo, utilizar técnicas anticipatorias como parte del tratamiento del dolor infantil conforme a su edad, cognitivo conductuales (1), o la distracción una técnica prometedora (7).

Respecto a capacitación, primero es precisa la formación constante de las Enfermeras en el manejo del dolor $(8,10)$, y segundo, el rol primordial de las Enfermeras en capacitación y apoyo a los padres frente a los procesos dolorosos, junto con la presencia del equipo (5). Para el cuidado 
humanizado se precisa de la integración de intervenciones no farmacológicas como una práctica efectiva para calmar el dolor y la ansiedad asociada a este, donde se considere la contención emocional y física, además del acercamiento familiar.

En este aspecto se considera el cuidado humanizado como pilar fundamental de la disciplina de enfermería, entendiéndose este como la consideración de la persona holística, que permita un manejo centrado en sus particularidades y que no se limite a un tratamiento convencional. Al igual que plantean las entrevistadas de este estudio, para desarrollar dichas habilidades, es necesario el autoconocimiento, y que la enfermera se nutra constantemente de su experiencia profesional, comprendiendo la salud-enfermedad y experiencia humana como un proceso de cuidado transpersonal, posicionando a la Enfermería como algo más profundo que la curación de una enfermedad (21). Dicha concepción del cuidado humanizado es compartida por un estudio realizado en Colombia (32), donde es posible identificar una construcción del concepto como aquel que concibe integralmente al individuo, familia y colectividad; reconociendo la dignidad y condición humana, reflexionando acerca de sus necesidades de cuidado en salud y de sus respuestas y promoviendo la recuperación a través de una comunicación asertiva y efectiva.

Como principal limitación, el estudio solo se centró en un único hospital, con una población de estudio completamente femenina, además de ser personas jóvenes, con una importante parte de la muestra en su primera experiencia laboral que puede influir en los resultados.

\section{Conclusiones}

En conclusión, se logra conocer la percepción de las enfermeras sobre el manejo del dolor en el Servicio de Oncología del HNRR, comprendiendo los niños y niñas como un ser multifactorial, que requiere una valoración integral que considere aspectos del dolor como intensidad, frecuencia, tipo, irradiación; y factores propios del niño, integrando la visión de padres y personal de salud.

Los resultados de esta investigación contribuyen para iniciar la construcción de estrategias para mejorar el manejo del dolor en niños por Enfermeras y Enfermeros, y su competencia específica en esta área; así poder desarrollar evidencia disciplinar que permita disminuir la brecha actual que se presenta en el manejo del dolor en niños.

\section{Referencias Bibliográficas}

1. Bárcena Fernández E. Manejo del dolor pediátrico en el centro de salud. Pediatría Atención Primaria [Internet]. 2014 [citado 2 de septiembre de 2020];16:37-43. Disponible en: http://scielo.isciii.es/pdf/pap/v16s23/foro2.pdf

2. Rodríguez Hernández I, Fajardo Villarroel A, Peña Jiménez M. Rol del enfermero en las etapas de evaluación, valoración e intervención del dolor en niños. Revista Habanera de Ciencias Médicas [Internet]. 2016 [citado 2 de septiembre de 2020];15(3):399-407. Disponible en: https://www.medigraphic.com/pdfs/revhabciemed/hcm-2016/hcm163i.pdf

3. Villacres S, Chumpitazi CE. Acute pediatric pain management in the primary care office. Pediatric annals [Internet]. 2018 [citado 2 de septiembre 2020];47(3):e124-e129. Disponible en: https://search.proquest.com/docview/2013697894/fulltextPDF/4514CC5A07194242PQ/1 ?accou ntid=14621

4. Gallego Muñoz C, Martínez Bautista MJ, Guerrero Navarro N, Romero Hernández I, García Martín F, Manzano Martín MV. Manejo del dolor en el paciente oncológico pediátrico y 
factores que influyen en su percepción. Revista Cubana de Farmacia [Internet]. 2015 [citado 2 de septiembre 2020];49(2):394-411. Disponible en: https://www.medigraphic.com/pdfs/revcubfar/rcf-2015/rcf152q.pdf

5. Aziznejadroshan P, Alhani F, Mohammadi E. Experiences of Iranian nurses on the facilitators of pain management in children: a qualitative study. Pain research and treatment [Internet]. 2016 [citado 2 de septiembre 2020];2016. Disponible en: downloads.hindawi.com/journals/prt/2016/3594240.pdf

6. Mediani HS, Duggan R, Chapman R, Hutton A, Shields L. An exploration of Indonesian nurses' perceptions of barriers to paediatric pain management. Journal of Child Health Care [Internet]. 2017 [citado 2 de septiembre 2020];21(3):273-282. Disponible en: https://journals.sagepub.com/doi/pdf/10.1177/1367493517715146

7. Bukola IM, Paula D. The effectiveness of distraction as procedural pain management technique in pediatric oncology patients: a meta-analysis and systematic review. Journal of pain and symptom management [Internet]. 2017 [citado 2 de septiembre 2020];54(4):589-600. Disponible en: https://www.jpsmjournal.com/article/S0885-3924(17)30269-5/fulltext

8. Notejane M, Bernadá M, Ruiz V, Klisich V. Knowledge of nursing staff at a pediatric referral hospital regarding the assessment and management of pain in children. Boletín médico del Hospital Infantil de México [Internet]. 2019 [citado 2 de septiembre 2020];76(1):24-30. Disponible

en: https://www.researchgate.net/profile/Martin_Notejane/publication/336914635_Knowledge_of_n ursing_staff_at_a_pediatric_referral_hospital_regarding_the_assessment_and_management_of_p ain_in_children/links/5dd34ff74585156b351e92f1/Knowledge-of-nursing-staff-at-a-pediatricreferral-hospital-regarding-the-assessment-and-management-of-pain-in-children.pdf 9. Boles J. Non-pharmacological strategies for addressing infant pain. Pediatr Nurs [Internet]. 2017 [citado 2 de septiembre de 2020];43(2):98-100. Disponible en: https://www.researchgate.net/profile/Jessika_Boles2/publication/322917507_NonPharmacological_Strategies_for_Addressing_Infant_Pain/links/5b8d4a1692851c6b7eb8f1eb/No n-Pharmacological-Strategies-for-Addressing-Infant-Pain.pdf

10. Peirce D, Corkish V, Lane M, Wilson S. Nurses' knowledge and attitudes regarding pediatric pain management in western Australia. Pain Management Nursing [Internet]. 2018 [citado 2 de septiembre de 2020];19(6):707-717. Disponible en: https://www.sciencedirect.com/science/article/abs/pii/S1524904217302576

11. Zisk-Rony RY, Lev J, Haviv H. Nurses' report of in-hospital pediatric pain assessment: Examining challenges and perspectives. Pain Management Nursing. 2015;16(2):112-120.

12. Simons J. A proposed model of the effective management of children's pain. Pain Management Nursing [Internet]. 2015 [citado 2 de septiembre 2020];16(4):570-578. Disponible en:

https://www.sciencedirect.com/science/article/pii/S1524904214001969?casa_token=z4XyPcjoa VEAAAAA:-FRi6Xg1IIm8OclZDBNw9WCSMyBObgXLay0td9AhGejDOOW_GSzt2KnnCIsvZOzmNET5So wUgs

13. Urtubia BF, Bravo AT, Zamora NR, Torres CP, Barria LC. Uso de opiáceos en niños con cáncer avanzado en cuidados paliativos. Revista chilena de pediatría [Internet]. 2016 [citado 2 de septiembre 2020];87(2):96-101. Disponible

en: https://www.sciencedirect.com/science/article/pii/S0370410615002600 
14. Zunino C, Notejane M, Bernadá M, Rodríguez L, Vanoli N, Rojas M, et al. Dolor en niños y adolescentes hospitalizados en un centro de referencia. Revista chilena de pediatría [Internet]. 2018 [citado 2 de septiembre 2020];89(1):67-73. Disponible en: https://scielo.conicyt.cl/scielo.php?pid=S0370-41062018000100067\&script=sci_arttext

15. Batalha LMC, Fernandes AM, de Campos C, Costa AMPMP. Pain assessment in children with cancer: a systematic review/Avaliação da dor em crianças com cancro: uma revisão sistemática/Evaluación del dolor en niños con cáncer: una revisión sistemática. Revista de Enfermagem Referência [Internet]. 2015 [citado 2 de septiembre 2020];4(5):119. Disponible en: https://search.proquest.com/docview/1842836142?pq-origsite=gscholar

16. Breilh J. Una perspectiva emancipadora de la investigación e incidencia basada en la determinación social de la salud. Eibenschutz C, Tamez S, González R, Compiladores. ' Determinación social o determinantes sociales de la salud [Internet]. 2011 [citado 2 de septiembre 2020];45-69. Disponible en: http://saludcolectiva-unr.com.ar/docs/SeminarioBreilh03.pdf

17. Collado Madurga AM, Odales Ibarra R, Piñón Gómez A, Alerm González A, González Pérez U, Acosta Quintana L. El dolor infantil, un acercamiento a la problemática desde la bioética. Revista Cubana de Pediatría [Internet]. 2012 [citado 2 de septiembre 2020];84(3):275-281. Disponible en: http://scielo.sld.cu/scielo.php?pid=S0034$75312012000300008 \&$ script=sci_arttext\&tlng=pt

18. Organización Mundial de la Salud 2020. Situación de la enfermería en el mundo 2020. Resumen de orientación. [Internet]. [citado 2 de septiembre 2020]. Disponible en: https://apps.who.int/iris/bitstream/handle/10665/331675/9789240003392-spa.pdf

19. Canales-Vergara M, Valenzuela-Suazo S, Paravic-Klijn T. Condiciones de trabajo de los profesionales de enfermería en Chile. Enfermería universitaria [Internet]. 2016 [citado 2 de septiembre 2020];13(3):178-186. Disponible en: http://www.scielo.org.mx/scielo.php?pid=S1665-70632016000300178\&script=sci_arttext

20. Bernadá M. Directrices de la OMS sobre el tratamiento farmacológico del dolor persistente en niños con enfermedades médicas. Archivos de Pediatría del Uruguay [Internet]. 2013 [citado 2 de septiembre 2020];84(2):143-145. Disponible en: http://www.scielo.edu.uy/pdf/adp/v84n2/v84n2a11.pdf

21. Urra E, Jana A, García M. Algunos aspectos esenciales del pensamiento de Jean Watson y su teoría de cuidados transpersonales. Ciencia y enfermería [Internet]. 2011 [citado 2 de septiembre 2020];17(3):11-22. Disponible en: https://scielo.conicyt.cl/pdf/cienf/v17n3/art02.pdf 22. Prieto CL, Galán CR, Kiza AH. Comparación de los conocimientos sobre dolor infantil en 2 poblaciones de profesionales de enfermería. En: Anales de Pediatría [Internet]. Elsevier; 2015 [citado 2 de septiembre de 2020]. p. e158-e164. Disponible en: https://www.analesdepediatria.org/es-comparacion-conocimientos-sobre-dolor-infantil-articuloS169540331400085X

23. Watson J. Watson s theory of human caring and subjective living experiences: carative factors/caritas processes as a disciplinary guide to the professional nursing practice. Texto \& Contexto-Enfermagem [Internet]. 2007 [citado 2 de septiembre 2020];16(1):129-135. Disponible en: http://www.scielo.br/scielo.php?pid=S0104-07072007000100016\&script=sci_arttext

24. Bardin L. Análise de Conteúdo. São Paulo: Edições 70; 2011.

25. Guba E, Lincoln Y. Competencia de paradigmas en la investigación cualitativa. Handbook of Qualitative Research. California: Sage Publications. 1994. 
26. Dos Santos FC, Camelo SHH, Laus AM, Leal LA. El enfermero de unidades hospitalarias oncológicas: perfil y capacitación profesional. Enfermería global [Internet]. 2015 [citado 2 de septiembre 2020];14(38):301-312. Disponible en:

http://scielo.isciii.es/pdf/eg/v14n38/revision3.pdf

27. Merskey, H. , Bogduk, N. Part III: Pain Terms, A Current List with Definitions and Notes on Usage. Classification of Chronic Pain. Second Edition, 209-214. IASP Press: Seattle.; 1994.

28. Chávez-Cañas WO, Becerra-Cristancho CP, Naranjo-García AS. Factores que intervienen en el manejo del dolor oncológico: un reto para el profesional de enfermería. MedUNAB [Internet]. 2016 [citado 2 de septiembre 2020];19(1):18-24. Disponible en: https://revistas.unab.edu.co/index.php/medunab/article/download/2211/2220

29. Moraes M, Zunino C, Duarte V, Ponte C, Favaro V, Bentancor S, et al. Evaluación de dolor en niños hospitalizados en servicios de salud públicos y privados de Uruguay. Archivos de Pediatría del Uruguay [Internet]. 2016 [citado 2 de septiembre de 2020];87(3):198-209. Disponible en: http://www.scielo.edu.uy/scielo.php?pid=s168812492016000300002\&script=sci_arttext

30. Notejane M, Le Pera V, Bernadá M. Conocimientos relativos al abordaje del dolor en niños: Encuesta a posgrados y residentes. Archivos de Pediatría del Uruguay [Internet]. 2016 [citado 2 de septiembre de 2020];87(4):323-331. Disponible en: http://www.scielo.edu.uy/scielo.php?pid=S1688-

$12492016000400004 \&$ script=sci_arttext\&tlng=en

31. Organization WH. WHO model list of essential medicines for children: 5th list, April 2015. 2015 [citado 2 de septiembre de 2020]; Disponible en:

https://www.who.int/medicines/publications/essentialmedicines/EMLc_2015_FINAL_amended_ AUG2015.pdf?ua=1

32. Espinosa Aranzales Á, Enríquez Guerrero C, Leiva Aranzalez F, López Arévalo M, Castañeda Rodríguez L. Construcción colectiva de un concepto de cuidado humanizado en enfermería. Ciencia y enfermería [Internet]. 2015 [citado 2 de septiembre 2020];21(2):39-49. Disponible en: https://scielo.conicyt.cl/scielo.php?pid=S071795532015000200005\&script=sci_arttext\&tlng=p

Cómo citar: Parra Giordano D, Cuadro Maturana V, Nies Moraga R, Pávez Castro C, Romero Torres M, Seoane Cabezas C. Percepción de las Enfermeras frente al manejo del dolor en niños en un Servicio Oncológico Hospitalario en Chile. Enfermería: Cuidados Humanizados. 2020; 9(2): 85-99. Doi: https://doi.org/10.22235/ech.v9i2.2094

Contribución de los autores: a) Concepción y diseño del trabajo, b) Adquisición de datos, c) Análisis e interpretación de datos, d) Redacción del manuscrito, e) Revisión crítica del manuscrito. D.P.R. ha contribuido en a,b,c,d,e; V.C.M. en a,b,c,d,e, R.N.M. en a,b,c,d,e; C.P.C. en a,b,c,d,e; M.R.T. en a,b,c,d,e; C.S.C. en a,b,c,d,e.

Editora científica responsable: Dra. Natalie Figueredo 